\title{
Control borroso de la remoción de estrógenos en un biorreactor de membranas
}

\section{Fuzzy control of the removal of estrogen in a membrane bioreactor}

\author{
Leadina Sánchez Barboza \\ Universidad Politécnica Salesiana Ecuador \\ lsanchezb@ups.edu.ec. \\ Ennodio Torres Cruz \\ Universidad Experimental Politécnica "Antonio José de Sucre" \\ ennodiotorrescruz@gmail.com
}

Recibido: 2 de julio 2016

Aceptado: 8 de agosto 2016

\begin{abstract}
Resumen
El Biorreactor de Membranas (BRM) emergió recientemente como un importante producto tecnológico para el tratamiento de aguas residuales que contienen contaminantes de tipo estrogénico, obteniendo efluentes de alta calidad. Debido a la naturaleza recalcitrante de los estrógenos, uno de los parámetros que se ha determinado como influyente en la remoción de estas sustancias es el Tiempo de Retención de Sólidos (TRS), ya que este permite un mayor tiempo de permanencia de la biomasa en el reactor. Se simuló en el Fuzzy Logic Toolbox de MATLAB la influencia del TRS en la remoción de estrógenos mediante control borroso; se borrosificaron los valores obtenidos por expertos en experimentos a escala de laboratorio, y sobre la base de las reglas de inferencia previamente diseñadas se realizó el proceso de inferencia borrosa, y finalmente se desborrosificó la salida para obtener de nuevo un valor nítido. El sistema de control borroso diseñado produjo muy buenos resultados, con porcentajes de error muy pequeños para la mayoría de los casos, excepto para la remoción de EE2 en el reactor con largo TRS. La simulación permite concluir que el Fuzzy Logic Toolbox es una buena herramienta para obtener resultados cercanos a los obtenidos mediante un sistema experimental real.
\end{abstract}

\section{Palabras clave}

Estrógeno, tiempo de retención de sólidos, biorreactor de membranas, control borroso, MATLAB.

\begin{abstract}
The Membrane Bioreactor (MBR) has recently emerged as an important technology product for the treatment of wastewater containing estrogens and contaminants and is capable of transforming a residual water in a high quality effluent. Because of the recalcitrant nature of both natural and synthetic estrogens, one of the parameters that has been determined as influential to the removal of these substances is the Solids Retention Time (SRT), as this allows more time spent in the biomass in the reactor. The influence of the SRT in estrogen removal was simulated in
\end{abstract}


the MATLAB Fuzzy Logic Toolbox using fuzzy control. For this purpose, the values measured or obtained by experts in laboratory scale experiments were fuzzified, and on the basis of previously designed inference rules, the inference process was performed, and finally the output is again desfuzzified for crisp value. The designed fuzzy control system produced very good results, with very small percentages of error for most cases, except for the removal of EE2 in the reactor with long SRT. The performance of the simulation allows us to conclude that the Fuzzy Logic Toolbox is a good tool to get close to the results obtained by an actual experimental system.

\section{Keywords}

Estrogen, solids retention time, membrane bioreactor, fuzzy control, MATLAB. 


\section{INTRODUCCIÓN}

Los estrógenos son compuestos de disrupción endocrina y pueden ser naturales como la estrona (E1), 17- $\beta$-estradiol (E2) y el estriol (E3), o sintéticos, como la 17- $\alpha$-etinilestradiol (EE2). Los estrógenos se excretan en mayor proporción por el sistema renal $\mathrm{y}$, aproximadamente, en una quinta parte por la bilis, llegando de esta manera a los recursos hídricos. De tal manera, en el tabla 1 se muestran las concentraciones medidas cuantitativamente en las aguas residuales municipales (1-2), en la entradas a las Plantas de Tratamiento de Aguas Residuales (PTAR) (3-6).

Tabla 1. Concentraciones de estrógenos en recursos hídricos.

\begin{tabular}{ccc}
\hline Recurso hídrico & Concentración (ng/L) & Referencia \\
\hline Efluente de una PTAR & 64 & Ternes, et al. (3) \\
Residual domestica & 150 & Vethaak, et al. (7) \\
Influente a una PTAR & 115 & Petrovic, et al. (9) \\
& 15.6 & Servos, et al. (5) \\
\hline
\end{tabular}

Sin embargo, los efectos endocrinos de los estrógenos se manifiestan a una concentración mucho más baja que la encontrada en las aguas residuales, siendo $0,1 \mathrm{ng}$ EE2/L (equivalente a $0.0001 \mu \mathrm{g}$ EE2/L). Algunos de los efectos endocrinos son por ejemplo: la feminización de los peces (8-11), el cáncer de mama (12), y los trastornos tiroideos (13-14).

Debido a que las concentraciones en los recursos hídricos son superiores a la concentración donde se manifiestan sus efectos, el interés y la necesidad de la investigación giran en torno a la remoción de compuestos estrogénicos (RCE). Los mecanismos de remoción de estrógenos del agua residual son la adsorción y la biodegradación (1516), los cuales están influenciados por numerosos factores, entre los que se encuentran las condiciones locales, el tipo de hormona y el proceso tecnológico aplicado para su depuración. El BRM emergió recientemente como un importante producto tecnológico para el tratamiento de agua residual AR, siendo capaz de transformar un agua residual en un efluente de alta calidad. En la Fig. 1 se observa la representación esquemática del BRM, el cual resulta de la combinación del sistema convencional de lodos activados con una unidad de membrana. En este sistema, la biomasa suspendida (medida en sólidos suspendidos totales en el licor mezcla, SSLM, o sólidos suspendidos volátiles en el licor mezcla, SSVLM) degrada los compuestos presentes en el influente, mientras que la unidad de membrana separa la biomasa del agua ya tratada.

Aunque el BRM tiene su origen en el sistema convencional de lodos activados, el tiempo de retención hidráulico, TRH (tiempo teórico de permanencia del influente en el reactor en contacto con la biomasa), está completamente disociado del TRS (tiempo promedio que la biomasa permanece el proceso de tratamiento), lo que permite una 
mayor flexibilidad en el control de los parámetros operacionales (17). En tal sentido, en un MBR se pueden alcanzar tiempos de retención de sólidos elevados de hasta de 50-60 días (18). Esto es importante para facilitar el desarrollo de microorganismos de crecimiento lento (17) como las bacterias nitrificantes (19), las cuales producen protoplasma, es decir, otras bacterias (20) que degradan sustancias complejas y de baja biodegradabilidad (17). Este es el caso de los compuestos estrogénicos, como por ejemplo las hormonas esteroideas. La remoción de estrógenos empleando un BRM ha sido reportada por muchos autores (16, 21-26). Así, el BRM es ejemplo de un sistema de tratamiento de agua capaz de remover sustancias estrogénicas presentes en aguas residuales. Los porcentajes de remoción de los compuestos estrogénicos (RCE) en este tipo de reactores dependen de varios factores entre los que destacan el TRS, la nitrificación y la actividad microbiana (26).

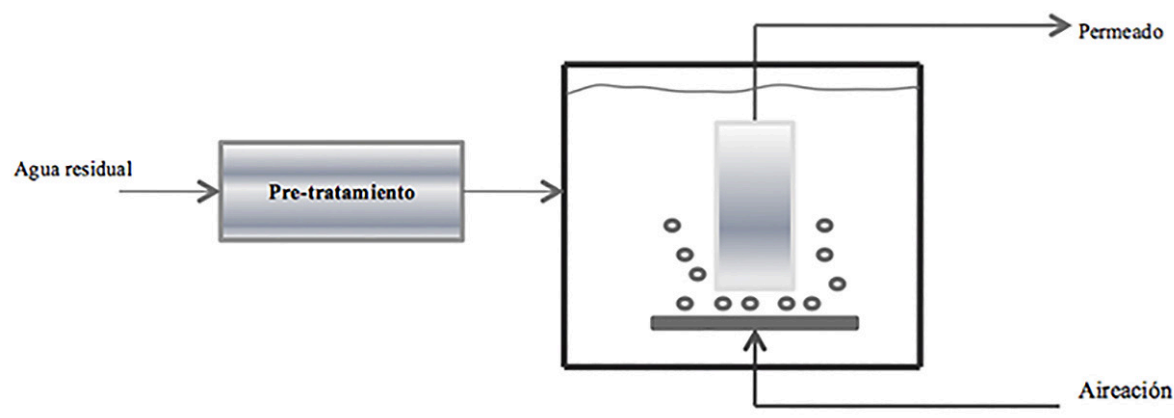

Figura 1. Biorreactor de Membranas

En función de lo planteado anteriormente, el objetivo de este trabajo es simular la influencia del TRS en la remoción de estrógenos mediante lógica borrosa. Para tales efectos, la metodología consistió en construir los conjuntos borrosos para la entrada (TRS) y salida (RCE) y, para formular las reglas borrosas, se tomó como base los resultados (nítidos) del trabajo de Maeng, et al. (26).

\section{CARACTERIZACIÓN DEL AGUA RESIDUAL QUE CONTIENE LOS ESTRÓGENOS}

La calidad del influente y del efluente se mide respecto a la demanda química de oxígeno (DQO), carbono orgánico disuelto (COD), la concentración de nutrientes (Nitrógeno, $\mathrm{N}$ y Fosforo, $\mathrm{P}$ ), el pH y el oxígeno disuelto (OD). En el tabla 2 se muestran las características del agua residual que contenía los estrógenos E1, E2 y EE2 utilizada por Maeng, et al. (26)

En la tabla anterior se observa que, independientemente del TRS, la materia orgánica presente disminuye a niveles prácticamente iguales y que el OD se mantiene constante en los tres reactores R1, R2 y R3, lo cual favorece la nitrificación, esto evidenciado por el consumo total de amonio $\left(\mathrm{NH}_{3}\right)$. Maeng, et al. (26) reportaron que no se observaron diferencias en la calidad del permeado, pero que la actividad microbiana específica de la biomasa fue diferente para R1, R2 y R3, decreciendo a medida que incrementaba el TRS. 
Tabla 2. Características del influente-efluente en los reactores R1, R2 y R3.

\begin{tabular}{|c|c|c|c|c|}
\hline Parámetro & Influente & $\begin{array}{c}\text { Efluente R1 } \\
\text { (TRS } 8 \mathrm{~d} \text { ) }\end{array}$ & $\begin{array}{l}\text { Efluente R2 } \\
\text { (TRS 20 d) }\end{array}$ & $\begin{array}{l}\text { Efluente R3 } \\
\text { (TRS } 80 \text { d) }\end{array}$ \\
\hline $\begin{array}{c}\text { DQO } \\
(\mathrm{mg} / \mathrm{L})^{\mathrm{b}}\end{array}$ & $449 \pm 3,4$ & $5,5 \pm 0,2$ & $5,7 \pm 0,4$ & $5,6 \pm 0,3$ \\
\hline $\begin{array}{l}\mathrm{COD} \\
(\mathrm{mg} / \mathrm{L})^{\mathrm{b}}\end{array}$ & $81 \pm 1,5$ & $1,8 \pm 0,2$ & $1,7 \pm 0,2$ & $1,3 \pm 0,1$ \\
\hline $\begin{array}{c}\mathrm{N}-\mathrm{T} \\
(\mathrm{mg} / \mathrm{L})^{\mathrm{b}}\end{array}$ & $44 \pm 1,0$ & $37,3 \pm 1,1$ & $39,1 \pm 0,7$ & $41,2 \pm 0,6$ \\
\hline $\begin{array}{l}\mathrm{NH}_{3}-\mathrm{N} \\
(\mathrm{mg} / \mathrm{L})^{\mathrm{b}}\end{array}$ & $20 \pm 0,1$ & N.D. & N.D. & N.D \\
\hline $\begin{array}{l}\mathrm{NO}_{3}-\mathrm{N} \\
(\mathrm{mg} / \mathrm{L})^{\mathrm{b}}\end{array}$ & N.D. & $36,5 \pm 2,1$ & $37,6 \pm 0,9$ & $41,2 \pm 0,7$ \\
\hline $\begin{array}{l}\mathrm{NO}_{2}-\mathrm{N} \\
(\mathrm{mg} / \mathrm{L})^{\mathrm{b}}\end{array}$ & N.D. & $0,2 \pm 0,04$ & $0,2 \pm 0,02$ & $0,2 \pm 0,04$ \\
\hline $\begin{array}{c}\mathrm{P}-\mathrm{T} \\
(\mathrm{mg} / \mathrm{L})^{\mathrm{b}}\end{array}$ & $6 \pm 0,7$ & $4,9 \pm 0,1$ & $5,6 \pm 0,4$ & $5,9 \pm 0,2$ \\
\hline $\begin{array}{l}\mathrm{PO}_{4}^{-\mathrm{T}} \\
(\mathrm{mg} / \mathrm{L})^{\mathrm{b}}\end{array}$ & $4 \pm 0,5$ & $4,6 \pm 0,2$ & $5,3 \pm 0,1$ & $5,7 \pm 0,2$ \\
\hline $\mathrm{PH}^{\mathrm{a}}$ & $7,7 \pm 0,1$ & $7,9 \pm 0,1$ & $7,9 \pm 0,1$ & $8,0 \pm 0,1$ \\
\hline $\mathrm{OD}(\mathrm{mg} / \mathrm{L})^{\mathrm{a}}$ & $7,1 \pm 0,2$ & $8,0 \pm 0,1$ & $8,0 \pm 0,1$ & $8,0 \pm 0,1$ \\
\hline
\end{tabular}

${ }^{\mathrm{a}} \mathrm{n}=180 ;{ }^{\mathrm{b}} \mathrm{n}=48 ;$ N.D. $=$ No detectado.

Fuente: Maeng, et al. (26).

\section{PORCENTAJES DE REMOCIÓN DE ESTRÓGENOS EN VARIOS BRM}

En el tabla 3 se muestran las concentraciones de los estrógenos removidos por Maeng, et al. (26) y los porcentajes de remoción individual de E1, E2 y EE2 en cada reactor, así como la remoción global de los tres estrógenos en cada reactor. Como se ha mencionado, para estudiar el efecto del TRS en la remociónde estrógenos, Maeng, et al. (26) utilizaron tres BRM con TRS distintos $(\mathrm{R} 1=8 \mathrm{~d}$; R2 $=20 \mathrm{~d}$; R3 = $80 \mathrm{~d})$.

Es de notar que los resultados obtenidos por Maeng, et al. (26) son nítidos, y, a fin de aplicar lógica difusa para simular la influencia del TRS, es necesario borrosificar los conjuntos de entrada y de salida, esto mediante variables linguísticas. Posteriormente, y a partir de la borrosificación realizada, se construyen las reglas de inferencia. 
Tabla 3. Concentraciones de E1, E2 y EE2 en el influente y efluente, RCE individuales y global en cada BRM.

\begin{tabular}{|c|c|c|c|c|c|c|c|c|}
\hline BRM & $\begin{array}{l}\text { Conc. } \\
(\mu \mathrm{g} / \mathrm{L})\end{array}$ & E1 & $\begin{array}{c}\text { RCE, } \\
\text { E1 (\%) }\end{array}$ & E2 & $\begin{array}{l}\text { RCE, } \\
\text { E2 (\%) }\end{array}$ & EE2 & $\begin{array}{c}\text { RCE, } \\
\text { EE2 } \\
(\%)\end{array}$ & $\begin{array}{c}\text { RCE } \\
(\%) \\
\text { gbal }\end{array}$ \\
\hline \multirow[b]{2}{*}{ R1 } & Inf & $0,97 \pm 0,01$ & \multirow{2}{*}{97.94} & $0,93 \pm 0,02$ & \multirow{2}{*}{97.94} & $0,70 \pm 0,03$ & \multirow[b]{2}{*}{39.50} & \multirow[b]{2}{*}{82.30} \\
\hline & Efl & $0,02 \pm 0,00$ & & $0,02 \pm 0,00$ & & $0,42 \pm 0,07$ & & \\
\hline \multirow{2}{*}{$\mathrm{R} 2$} & Inf & $0,97 \pm 0,01$ & \multirow{2}{*}{100.00} & $0,93 \pm 0,02$ & \multirow{2}{*}{100.00} & $0,70 \pm 0,03$ & \multirow{2}{*}{70.60} & \multirow{2}{*}{91.92} \\
\hline & Efl & $0,00 \pm 0,00$ & & $0,00 \pm 0,00$ & & $0,21 \pm 0,05$ & & \\
\hline \multirow{2}{*}{ R3 } & Inf & $0,97 \pm 0,01$ & \multirow{2}{*}{100.00} & $0,70 \pm 0,03$ & \multirow{2}{*}{100.00} & $0,70 \pm 0,03$ & \multirow{2}{*}{78.10} & \multirow{2}{*}{94.23} \\
\hline & Efl & $0,00 \pm 0,00$ & & $0,15 \pm 0,03$ & & $0,15 \pm 0,03$ & & \\
\hline \multirow{2}{*}{$\begin{array}{l}\mathrm{R} 2+ \\
\mathrm{ATU}\end{array}$} & Inf & $0,95 \pm 0,01$ & \multirow{2}{*}{82.10} & $0,95 \pm 0,02$ & \multirow{2}{*}{89.47} & $0,82 \pm 0,02$ & \multirow{2}{*}{46.34} & \multirow{2}{*}{73.89} \\
\hline & Efl & $0,17 \pm 0,03$ & & $0,10 \pm 0,05$ & & $0,44 \pm 0,04$ & & \\
\hline \multirow{2}{*}{$\begin{array}{c}\mathrm{R} 3+ \\
\mathrm{NaN} 3\end{array}$} & Inf & $0,95 \pm 0,01$ & \multirow{2}{*}{33.68} & $0,82 \pm 0,02$ & \multirow{2}{*}{12.63} & $0,82 \pm 0,02$ & \multirow{2}{*}{7.32} & \multirow{2}{*}{18.38} \\
\hline & Efl & $0,63 \pm 0,02$ & & $0,76 \pm 0,03$ & & $0,76 \pm 0,03$ & & \\
\hline
\end{tabular}

Conc: concentración, Inf: influente, Efl: efluente.

Fuente: Maeng, et al. $(26)(\mathrm{n}=24)$

\section{LÓGICA BORROSA}

En esta sección se comienza con las definiciones de proposición booleana y de proposición borrosa (tabla 4), las cuales se presentan en forma paralela con el propósito de destacar que la segunda es una generalización de la primera. En sentido pedagógico, se han elaborado ambas definiciones con un contenido inicial idéntico y un contenido final que refleja la generalización construida.

Tabla 4. Definiciones de proposición booleana y de proposición borrosa

\begin{tabular}{cc}
\hline Proposición booleana & Proposición borrosa \\
\hline $\begin{array}{c}\text { Secuencia finita de letras, palabras, símbolos, signos o caracteres, cuyo sentido gramatical completo es } \\
\text { susceptible de poseer una cualidad lógica bivalente (polivalente): }\end{array}$ \\
$\begin{array}{c}\text { pe es verdadera o es falsa; que en valor numérico corres- } \\
\text { ponde o al } 1 \text { o al } 0 .\end{array}$ & $\begin{array}{c}\text { grado de verdad expresado con un número } \\
\text { real en el intervalo } \mathrm{I}=\{\mathrm{x} \in \mathrm{R}: 0 \leq \mathrm{x} \geq 1\} .\end{array}$ \\
\hline
\end{tabular}

El universo del discurso de las proposiciones booleanas se representa con la letra B, inicial del apellido del matemático británico George Boole (1815-1864), quien desarrolló los principios fundamentales del álgebra de la lógica con dos grados de verdad $\{0,1\}$. 
El universo del discurso de las proposiciones borrosas se denota con la letra L, inicial del apellido del lógico y matemático polaco Jan Lukasiewicz (1878-1956), quien trata por primera vez en 1920 la lógica trivalente, es decir, una lógica con tres grados de verdad $\{0,1 / 2,1\}$ que fue generalizada en 1921 por el matemático estadounidense de origen polaco Emil Post (1897-1954) a una lógica finita cualquiera; y esta, a su vez, fue extendida a un número infinito de valores de verdad en un trabajo de investigación realizado por Lukasiewicz en colaboración con el lógico y matemático estadounidense de origen polaco Alfred Tarski (1902-1983) y publicado en 1930. Estas tres versiones fueron integradas en un modelo denominado lógica estándar, lógica polivalente o lógica de Lukasiewicz.

En este contexto, se tiene una función de veracidad V $: \mathrm{L} \rightarrow \mathrm{I}$, que a cada proposición borrosa $\mathrm{p} \epsilon \mathrm{L}$, asocia un único grado de verdad $\mathrm{V}(\mathrm{p}) \epsilon \mathrm{I}$, a la cual se le exige que su rango $\mathrm{V}(\mathrm{L})$ sea un superconjunto de $\{0,1\}$, es decir, $\{0,1\} \subseteq \mathrm{V}(\mathrm{L})$. Siendo así, se dice que la pareja $(\mathrm{L}, \mathrm{V})$ es una lógica borrosa, o simplemente una lógica.

El rasgo de distinción de una lógica borrosa reside en las formas como se definen sus operaciones fundamentales: negación, conjunción, disyunción, implicación, bicondicional, y disyunción exclusiva, que se designan con las constantes lógicas $\sim, \wedge, \vee, \Rightarrow>$, $\Leftrightarrow$, y $\nabla$, respectivamente (tabla 5 ).

Tabla 5. Definición de las operaciones fundamentales de la lógica borrosa

\begin{tabular}{cc}
\hline Notación & Lectura en español \\
\hline$\sim \mathrm{p}$ & La negación de $\mathrm{p}$ \\
$\mathrm{p} \wedge \mathrm{q}$ & $\mathrm{p} \mathrm{y} \mathrm{q}$ \\
$\mathrm{p} \vee \mathrm{q}$ & $\mathrm{p} \mathrm{o} \mathrm{q}$ \\
$\mathrm{p}=>\mathrm{q}$ & Si p, entonces $\mathrm{q}$ \\
$\mathrm{p} \Leftrightarrow \mathrm{q}$ & $\mathrm{p}$ si y sólo si q \\
$\mathrm{p} \nabla \mathrm{q}$ & O p o q \\
\hline
\end{tabular}

En los casos de la lógica booleana y de una lógica estándar, tales operaciones son definidas como se especifican en el tabla 6.

Sean p y q dos proposiciones borrosas en una lógica $(\mathrm{L}, \mathrm{V})$. Se dice que p y q son equivalentes si y solo si los grados de verdad de ambas proposiciones son iguales. Se denota $\mathrm{p} \equiv \mathrm{q}$. En forma compacta: $\mathrm{p} \equiv \mathrm{q} \Leftrightarrow \mathrm{V}(\mathrm{p})=\mathrm{V}(\mathrm{q})$.

La equivalencia en una lógica borrosa $(\mathrm{L}, \mathrm{V})$ satisface las propiedades reflexiva $[\mathrm{p}$ $\equiv \mathrm{p}]$, simétrica $[(\mathrm{p} \equiv \mathrm{q}) \Leftrightarrow(\mathrm{q} \equiv \mathrm{p})], \mathrm{y} \operatorname{transitiva}[(\mathrm{p} \equiv \mathrm{q}) \wedge(\mathrm{q} \equiv \mathrm{r})) \Rightarrow(\mathrm{p} \equiv \mathrm{r})]$

En el tabla 7 se colocan las caracterizaciones de conjunto cantoriano y de conjunto borroso en forma paralela, con el propósito de destacar que la teoría borrosa de conjuntos es una generalización de la teoría cantoriana de conjuntos; es decir, esta es un caso particular de aquella. Utilizamos la expresión "conjunto cantoriano" en honor al 
matemático ruso-alemán Georg Cantor (1845-1918) fundador de la teoría de conjuntos, teoría que representó un cambio de paradigma en las ciencias matemáticas pues generó una nueva corriente de pensamiento, denominada matemática moderna.

Tabla 6. Operaciones en $(\mathrm{B}, \sim, \wedge, \mathrm{v})$ y en $(\mathrm{L}, \sim, \wedge, \vee)$

\begin{tabular}{|c|c|c|}
\hline \multicolumn{2}{|c|}{ Nombre de la operación } & Definición en lenguaje matemático \\
\hline \multirow{2}{*}{ Negación } & Booleana & $\sim\left[\begin{array}{ll}0 & 1\end{array}\right] \stackrel{\text { def }}{=}\left[\begin{array}{ll}1 & 0\end{array}\right]$ \\
\hline & Estándar & $\mathrm{V}(\sim \mathrm{p}) \stackrel{\text { def }}{=} 1 \mathrm{~V}(\mathrm{p})$ \\
\hline \multirow{2}{*}{ Conjunción } & Booleana & {$\left[\begin{array}{llll}0 & 0 & 1 & 1\end{array}\right] \wedge\left[\begin{array}{llll}0 & 1 & 0 & 1\end{array}\right] \stackrel{\text { def }}{=}\left[\begin{array}{llll}0 & 0 & 0 & 1\end{array}\right]$} \\
\hline & Estándar & $\mathrm{V}(\mathrm{p} \wedge \mathrm{q}) \stackrel{\text { def }}{=} \operatorname{mín}\{\mathrm{V}(\mathrm{p}), \mathrm{V}(\mathrm{q})\}$ \\
\hline \multirow{2}{*}{ Disyunción } & Booleana & {$\left[\begin{array}{llll}0 & 0 & 1 & 1\end{array}\right] \vee\left[\begin{array}{llll}0 & 1 & 0 & 1\end{array}\right] \stackrel{\text { def }}{=}\left[\begin{array}{llll}0 & 1 & 1 & 1\end{array}\right]$} \\
\hline & Estándar & $\mathrm{V}(\mathrm{p} \vee \mathrm{q}) \stackrel{\text { def }}{=} \operatorname{máx}\{\mathrm{V}(\mathrm{p}), \mathrm{V}(\mathrm{q})\}$ \\
\hline \multirow{2}{*}{ Implicación } & Booleana & {$\left[\begin{array}{llll}0 & 0 & 1 & 1\end{array}\right]=>\left[\begin{array}{llll}0 & 1 & 0 & 1\end{array}\right] \stackrel{\text { def }}{=}\left[\begin{array}{llll}1 & 1 & 0 & 1\end{array}\right]$} \\
\hline & Estándar & $\mathrm{V}(\mathrm{p}=>\mathrm{q}) \stackrel{\text { def }}{=} \operatorname{mín}\{1,1 \mathrm{~V}(\mathrm{p}) \mathrm{V}(\mathrm{q})\}$ \\
\hline \multirow{2}{*}{ Bicondicional } & Booleano & {$\left[\begin{array}{llll}0 & 0 & 1 & 1\end{array}\right] \Leftrightarrow\left[\begin{array}{llll}0 & 1 & 0 & 1\end{array}\right] \stackrel{\text { def }}{=}\left[\begin{array}{llll}1 & 0 & 0 & 1\end{array}\right]$} \\
\hline & Estándar & $\mathrm{V}(\mathrm{p} \Leftrightarrow \mathrm{q}) \stackrel{\text { def }}{=} 1|\mathrm{~V}(\mathrm{p}) \mathrm{V}(\mathrm{q})|$ \\
\hline \multirow{2}{*}{$\begin{array}{l}\text { Disyunción } \\
\text { Exclusiva }\end{array}$} & Booleana & {$\left[\begin{array}{llll}0 & 0 & 1 & 1\end{array}\right] \nabla\left[\begin{array}{llll}0 & 1 & 0 & 1\end{array}\right] \stackrel{\text { def }}{=}\left[\begin{array}{llll}0 & 1 & 1 & 0\end{array}\right]$} \\
\hline & Estándar & $V(p \nabla q) \stackrel{\text { def }}{=}|V(p) V(q)|$ \\
\hline
\end{tabular}

Utilizamos la expresión “conjunto cantoriano" en honor al matemático ruso-alemán Georg Cantor (1845-1918) fundador de la teoría de conjuntos, teoría que representó un cambio de paradigma en las ciencias matemáticas pues generó una nueva corriente de pensamiento, denominada matemática moderna.

El tratamiento de un conjunto borroso a través de su función de pertenencia es una idea original de Zadeh, quien la hizo pública el año 1965 en su famoso artículo "Fuzzy Sets". Sus estudios sobre las lógicas polivalentes los realizó en el Instituto de Estudios Avanzados de Princeton y su facilitador más influyente fue el matemático estadounidense Stephen Cole Kleene (1909-1994). Además de ser el fundador de la teoría de conjuntos borrosos, Zadeh es la persona que ha iniciado las líneas de investigación fundamentales sobre el análisis de sistemas complejos y los procesos de decisión, la lógica borrosa y sus implicaciones en diversos dominios tales como el razonamiento aproximado, la gestión de la incertidumbre, los sistemas expertos, las redes neuronales, la información granular y su centralidad en el razonamiento humano, y la computación con palabras. Por sus excepcionales contribuciones, Zadeh es considerado el principal pionero en la fundación de la "Fuzzy Logic", la cual representa un cambio de paradigma en las ciencias matemáticas pues está generando una nueva corriente de pensamiento, que Torres (27) ha denominado matemática postmoderna. 
El rasgo de distinción de una teoría borrosa de conjuntos reside en las formas en las que se definen sus operaciones canónicas: complemento, intersección, y unión, que se designan con las constantes matemáticas ${ }^{-}, \cap, \mathrm{y} \cup$, respectivamente (tabla 8). Tanto las definiciones como las propiedades de tales operaciones matemáticas serán enunciadas utilizando conjuntos variables que designaremos con letras latinas mayúsculas en formato de cursiva A, B, C, D, etc., y con subíndices si fuera necesario.

Tabla 7. Características de conjunto cantoriano y de conjunto borroso

\begin{tabular}{cc}
\hline Conjunto cantoriano & Conjunto borroso \\
\hline A es un conjunto cantoriano si y sólo & Un conjunto borroso $A$ en el universo \\
si para cualquier elemento $x$, se tiene la & $X$ está caracterizado por su función de \\
disyunción & pertenencia $\mu_{\mathrm{A}}: X$ 政, es decir, por \\
exclusiva de las proposiciones & la correspondencia que a cualquier \\
booleanas & elemento $x \in X$, le asocia un único \\
$x \in A:$ " $x$ es un elemento de $A$ " y & número real $\mu_{\mathrm{A}}(x)$ que representa el \\
$x A:$ " $x$ no es un elemento de $A$ ", & grado de pertenencia del elemento $x$ \\
donde el símbolo ' $\epsilon$ ' denota la & al conjunto borroso $A$. \\
denominada relación de pertenencia. & \\
\hline
\end{tabular}

Tabla 8. Operaciones canónicas.

\begin{tabular}{lc}
\hline \multicolumn{1}{c}{ Notación } & Lectura en español \\
\hline $\bar{A}$ & El complemento de $A$ \\
$A \cap B$ & $A$ intersección $B$ \\
$A \cup B$ & $A$ unión $B$ \\
\hline
\end{tabular}

En los casos de las teorías cantoriana y estándar de conjuntos, tales operaciones se definen como se especifican en el tabla 9, donde $P(X)$ [respectivamente, $G(X)$ ] es la familia de conjuntos cantorianos [respectivamente, borrosos] en el universo X:

En el tabla 10 aparecen las propiedades que dotan al conjunto de las proposiciones borrosas y a la familia de los conjuntos borrosos en el universo X de una misma estructura algebraica, denominada reticulado de De Morgan.

Cada propiedad en $(\mathrm{L}, \sim, \wedge, \vee)$ fundamenta la respectiva propiedad en $\left(\mathrm{G}(\mathrm{X}),{ }^{-}, \cap\right.$, $\cup)$. El elemento neutro de la conjunción se representa con el número uno en negrita [1] y se denomina la proposición segura, pues tiene un valor lógico fijo 1. De manera análoga, el elemento neutro de la conjunción se representa con el número cero en negrita [0] y se denomina la proposición imposible, pues tiene un valor lógico fijo 0. Por lo tanto, las proposiciones segura e imposible son proposiciones booleanas constantes. Las proposiciones booleanas que son equivalentes a la proposición segura, son llamadas tautologías; las que son equivalentes a la proposición imposible, contradicciones. 
El elemento neutro de la intersección es el conjunto cantoriano X que juega el papel de universo de los conjuntos borrosos en consideración; en cambio, el elemento neutro de la unión es el conjunto vacío, representado por el símbolo " $\varnothing$ ".

Tabla 9. Operaciones en $\left(\mathrm{P}(\mathrm{X}),{ }^{-}, \cap, \cup\right)$ y en $\left(\mathrm{G}(\mathrm{X}),{ }^{-}, \cap, \cup\right)$

\begin{tabular}{ccc}
\hline \multicolumn{2}{c}{ Nombre de la operación } & \multicolumn{1}{c}{ Definición en lenguaje matemático } \\
\hline Complemento & Cantoriano & $\bar{A} \stackrel{\text { def }}{=}\{\mathrm{x} \in \mathrm{X}: \mathrm{x} \notin \mathrm{A}\}$ \\
& Estándar & $\bar{A} \stackrel{\text { def }}{=}\{\mathrm{x} \mid \mu \bar{A}(\mathrm{x}): \mu \bar{A}(\mathrm{x})=1-\mu \bar{A}(\mathrm{x})\}$ \\
\hline Intersección & Cantoriana & $A \cap B \stackrel{\text { def }}{=}\{\mathrm{x} \in \mathrm{X}: \mathrm{x} \in \mathrm{A} \wedge \mathrm{x} \in \mathrm{B}\}$ \\
& Estándar & $A \cap B \stackrel{\text { def }}{=}\left\{\mathrm{x} \mid \mu_{A \cap B}(\mathrm{x}): \mu_{A \cap B}(\mathrm{x})=\operatorname{mín}\left\{\mu_{\mathrm{A}}(\mathrm{x}), \mu_{\mathrm{B}}(\mathrm{x})\right\}\right.$ \\
\hline Unión & Cantoriana & $A \cup B \stackrel{\text { def }}{=}\{\mathrm{x} \in \mathrm{X}: \mathrm{x} \in \mathrm{A} \vee \mathrm{x} \in \mathrm{B}\}$ \\
& Estándar & $A \cup B \stackrel{\text { def }}{=}\left\{\mathrm{x} \mid \mu_{A \cup B}(\mathrm{x}): \mu_{A \cup B}(\mathrm{x})=\operatorname{máx}\left\{\mu_{\mathrm{A}}(\mathrm{x}), \mu_{\mathrm{B}}(\mathrm{x})\right\}\right.$ \\
\hline
\end{tabular}

Tabla 10. Reticulados $(\mathrm{L}, \sim, \wedge, \mathrm{v})$ y $(\mathrm{G}(\mathrm{X}),-, \cap, \mathrm{U})$

\begin{tabular}{|c|c|c|}
\hline Nombre & Propiedad en $(\mathrm{L}, \sim, \wedge, \vee)$ & Propiedad en $\left(\mathrm{G}(\mathrm{X}),{ }^{-},,\right)^{\prime}$ \\
\hline \multirow{2}{*}{ Conmutativa } & $(p \wedge q) \equiv(q \wedge p)$ & $\mathrm{A} \cap \mathrm{B}=\mathrm{B} \cap \mathrm{A}$ \\
\hline & $(p \vee q) \equiv(q \vee p)$ & $\mathrm{A} \cup \mathrm{B}=\mathrm{B} \cup \mathrm{A}$ \\
\hline \multirow{2}{*}{ Asociativa } & $((p \wedge q) \wedge r) \equiv(p \wedge(q \wedge r))$ & $(A \cap B) \cap C=A \cap(B \cap C)$ \\
\hline & $((p \vee q) \vee r) \equiv(p \vee(q \vee r))$ & $(A \cup B) \cup C=A \cup(B \cup C)$ \\
\hline \multirow{2}{*}{ Absorción } & $(p \wedge(p \vee q)) \equiv p$ & $A \cap(A \cup B)=A$ \\
\hline & $(p \vee(p \wedge q)) \equiv p$ & $\mathrm{~A} \cup(\mathrm{A} \cap \mathrm{B})=\mathrm{A}$ \\
\hline \multirow{2}{*}{ Distributiva } & $(p \wedge(q \vee r)) \equiv((p \wedge q) \vee(p \wedge r))$ & $\mathrm{A} \cap(\mathrm{B} \cup \mathrm{C})=(\mathrm{A} \cap \mathrm{B}) \cup(\mathrm{A} \cap \mathrm{C})$ \\
\hline & $(p \vee(q \wedge r)) \equiv((p \vee q) \wedge(p \vee r))$ & $\mathrm{A} \cup(\mathrm{B} \cap \mathrm{C})=(\mathrm{A} \cup \mathrm{B}) \cap(\mathrm{A} \cup \mathrm{C})$ \\
\hline \multirow{2}{*}{$\begin{array}{l}\text { Leyes de } \\
\text { De Morgan }\end{array}$} & $(\sim(p \wedge q)) \equiv((\sim p) \vee(\sim q))$ & $\overline{A \cap B}=\bar{A} \cup \bar{B}$ \\
\hline & $(\sim(p \vee q)) \equiv((\sim p) \wedge(\sim q))$ & $\overline{A \cup B}=\bar{A} \cap \bar{B}$ \\
\hline \multirow{2}{*}{$\begin{array}{l}\text { Elementos } \\
\text { Neutros }\end{array}$} & $(p \wedge \mathbf{1}) \equiv(\mathbf{1} \wedge p) \equiv p$ & $A \cap X=X \cap A=A$ \\
\hline & $(p \vee \mathbf{0}) \equiv(\mathbf{0} \vee p) \equiv p$ & $\mathrm{~A} \cup \varnothing=\varnothing \cup \mathrm{A}=\mathrm{A}$ \\
\hline
\end{tabular}

Las propiedades conmutativa, asociativa, absorción, distributiva y elementos neutros se colocaron emparejadas para resaltar la dualidad que existe entre la conjunción y la disyunción (respectivamente, la intersección y la unión); además, las leyes de De Morgan establecen un bucle recursivo entre ellas, a través de la negación (resp., el complemento); en consecuencia, los reticulados $(\mathrm{L}, \sim, \wedge, \vee)$ y $\left(\mathrm{G}(\mathrm{X}),{ }^{-}, \cap, \cup\right)$ son un ejemplos de unidualidad formal. 


\section{SISTEMA DE CONTROL BORROSO PARA LA REMOCIÓN DE ESTRÓGENOS}

El esquema general del razonamiento aproximado multicondicional tiene la forma:

$\begin{array}{ll}\text { Regla } 1 & \text { Si X es } A_{1} \text {, entonces Y es } B_{1} \\ \text { Regla } 2 & \text { Si X es } A_{2} \text {, entonces Y es } B_{2} \\ \quad \vdots & \vdots \\ \text { Regla } n & \text { Si X es } A n, \text { entonces Y es } B n \\ \text { Hecho } & \text { X es } H \\ \text { Conclusión } & \text { Y es } C\end{array}$

En este esquema,

$A_{1}, A_{2}, \ldots, A_{n}$ y $H$ son conjuntos borrosos en el universo finito $X$;

$B_{I}, B_{2}, \ldots, B_{n}$ y $C$ son conjuntos borrosos en el universo finito $Y$;

$X, Y$ son los conjuntos de los valores de las variables $\mathrm{X}, \mathrm{Y}$, respectivamente.

Paso 1. Para cada $j \in \mathrm{N}_{[1, n]}$, calcular el grado de consistencia, $r_{j}(\mathrm{H})$, entre el hecho dado y el antecedente de la j-ésima regla de inferencia si-entonces en términos de la altura de la intersección de los conjuntos borrosos asociados $\mathrm{H}$ y $\mathrm{A}_{\mathrm{j}}$. En lenguaje matemático:

$r_{j}(H)=\mu\left(H \cap A_{\mathrm{j}}\right)$,

o, usando la intersección estándar,

$\left.r_{j}(H)=\operatorname{máx}\left\{\operatorname{mín}\left\{\mu_{H}(x), \mu_{A j}\right)(x)\right\}: x \in X\right\}$

Paso 2. Para cada $j \in \mathrm{N}_{[1, n]}$, calcular el conjunto borroso $C$ truncando el conjunto borroso $B_{j}$ mediante el grado de consistencia $r_{j}(H)$, y tomando la unión estándar de los $n$ conjuntos borrosos truncados. En lenguaje matemático:

$$
\left.(\forall y \in Y)\left[\mu_{C}(y)=\operatorname{máx}\left\{\operatorname{mín}\left\{r_{j}(H), \mu_{B j}\right)(y)\right\}: j \in \mathrm{N}_{[1, n]}\right\}\right]
$$

El razonamiento aproximado multicondicional es utilizado en los controladores diseñados con base en la lógica borrosa. En general, un controlador borroso consiste en cuatro módulos:

- una base de reglas de inferencia,

- un motor de inferencia borrosa,

- un módulo de borrosificación, y

- un módulo de desborrosificación. 
Además, funciona repitiendo el ciclo de los cuatro pasos siguientes:

1. Las medidas son tomadas de todas las variables que representan las condiciones relevantes del proceso controlado.

2. Las medidas tomadas son convertidas en conjuntos borrosos apropiados para expresar medidas de incertidumbre. Este paso es llamado borrosificación.

3. Las medidas borrosificadas son usadas por el motor de inferencia para evaluar las reglas de control almacenadas en la base de reglas de inferencia. El resultado de esta evaluación es un conjunto borroso (o varios conjuntos borrosos) definido(s) en el (los) universo(s) de acciones posibles.

4. Este (estos) conjunto(s) borroso(s) es (son) convertido(s) en un solo número real (vector de números reales) que, en algún sentido, es el mejor representante del conjunto borroso (de los conjuntos borrosos). A esta conversión se le llama desborrosificación.

El control borroso de procesos encuentra numerosas aplicaciones en la industria, medicina, aeronáutica, electrónica, entre otros, e incluso en los últimos años, en el mundo de los aparatos electrodomésticos (28). Particularmente, el empleo del control borroso en plantas de tratamiento de aguas residuales abarca entre otras aplicaciones: la aireación en un proceso de lodos activados (29), la remoción de nitrógeno (30), el control del pH (31), el control de los sólidos en suspensión (32), el control predictivo de todo el proceso de tratamiento (33), el control del OD (34), el control anaeróbico (35), el control de flujo de metano para el tratamiento de aguas residuales vinícolas por digestión anaerobia (36), estudios de la capacidad de bioadsorción del fenol (37), el modelamiento matemático del control de proceso (38), simulación del proceso de nitrificación (39) y, más recientemente ha sido utilizado por Sánchez (40) para la valoración del impacto ambiental generado por contaminantes emergentes en aguas residuales hospitalarias.

Ahora bien, la estructura de un sistema de control borroso generalmente está basada en la monitorización de los valores de referencia del sistema (32). El sistema opera en dos niveles: el numérico y el lingüístico. Así, mientras el proceso controlado tiene que operar con valores numéricos reales, el sistema borroso tiene una configuración operacional con valores lingüísticos. Esto hace necesario que los valores numéricos reales de las medidas tomadas del proceso controlado sean transformados en términos cualitativos (por ejemplo: muy corto, corto, normal, bajo, alto) y asociados a ellos con determinado grado de pertenencia, de manera que se obtendrán números borrosos (32). Posteriormente, con la interacción de los expertos que conocen el proceso a controlar (para nuestro caso, los expertos son Maeng, et al. (26)) se construye un conjunto de reglas de inferencia, es decir, de reglas del tipo "Si X es A, entonces Y es B" que determinan la acción de control del sistema borroso en función de las variables de entrada (inferencia borrosa). Finalmente, la salida borrosa es transformada en un valor numérico real para que la acción de control pueda ejecutarse en el proceso. A continuación, en la Fig. 2 se muestra un esquema de la estructura del sistema de control borroso para la remoción de estrógenos en un BRM. 
En el tabla 11 se muestra la definición de las variables lingüísticas para los conjuntos borrosos de entrada (TRS) y salida (REC). Estas variables o términos lingüísticos permitirán construir las reglas borrosas del tipo " $\mathrm{Si}$, entonces", las cuales se presentan más adelante.

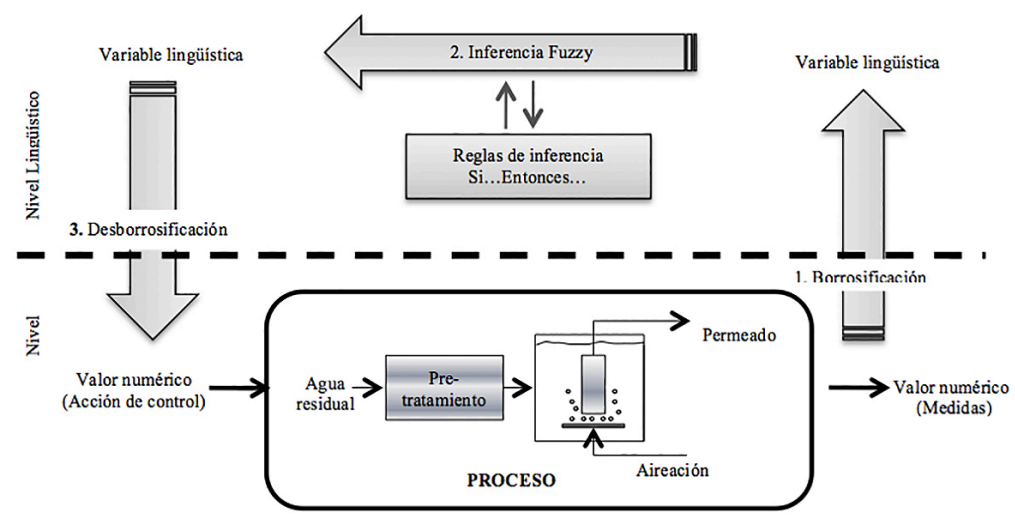

Figura 2. Estructura del sistema de control borroso para el proceso de remoción de estrógenos en el BRM

Tabla 11. Definición de variables lingüísticas de los conjuntos borrosos de entrada y salida

\begin{tabular}{ccc}
\hline Conjunto borroso & Rango & Variable lingüística \\
\hline Entrada & \\
\hline 6 a 8 & Muy Corto \\
10 a 20 & Corto \\
30 a 50 & Normal \\
60 a 80 & Largo \\
\hline TRS (d) & Salida & \\
\hline 5 a 10 & Insignificante \\
11 a 29 & Muy Baja \\
30 a 59 & Baja \\
60 a 79 & Media \\
80 a 90 & Considerable \\
91 a 98 & Alta \\
98,1 a 99,4 & Muy alta \\
99,5 a 100 & Óptima \\
\hline
\end{tabular}


Las reglas de inferencia borrosa definidas mediante la interacción con los resultados de Maeng, et al. (26) son las siguientes:

1. Si el TRS es muy corto, entonces la remoción de E1 es alta y la remoción de E2 es alta y la remoción de EE2 es muy baja.

2. Si el TRS es corto, entonces la remoción de E1 es óptima y la remoción de E2 es óptima y la remoción de EE2 es media.

3. Si el TRS es largo, entonces la remoción de E1 es óptima y la remoción de E2 es óptima y la remoción de EE2 es considerable.

\section{RESULTADOS Y DISCUSIÓN}

El propósito del modelo de control borroso diseñado e implementado es simular la interacción entre varios parámetros biológicos en la remoción de estrógenos en aguas residuales empleando BRM. Tal y como era de esperar, las entradas (TRS) determinan el comportamiento de las salidas (RCE). Para llevar a cabo la simulación se utilizó la herramienta Fuzzy Logic Toolbox de MATLAB. Las reglas de inferencia borrosa se diseñaron con base en los resultados del trabajo de Maeng, et al. (26), el cual se consideró como análogo a la opinión de expertos.

En el tabla 12 se muestra para cada reactor ( $\mathrm{R} 1, \mathrm{R} 2$ y $\mathrm{R} 3$ ) el contraste de los resultados obtenidos por Maeng, et al. (26) en el BRM y los obtenidos por el Fuzzy Logic Toolbox de MATLAB, y en la última columna se presenta el error entre las dos técnicas. Como es de notar, se consiguió el mismo porcentaje de remoción para E1 y E2 en R2 y R3, y también para EE2 en R1. En contraparte, la remoción obtenida por simulación de EE2 en R2 discordó muy ligeramente de la experimental, siendo su error relativo del 1,56\%. De la misma forma, la remoción de E1 y E2 en R1 fue ligeramente diferente entre la simulación y la realizada experimentalmente por Maeng, et al. (26) con errores relativos del 3,51 y 3,42\%, respectivamente. Sin embargo, un error relativo de $8,83 \%$ se presentó entre ambos métodos. El error se calculó tomando como patrón el valor obtenido experimentalmente por Maeng, et al. (26), y se realizó con la siguiente expresión: \%Error = [(Maeng - Fuzzy Logic) $/(\text { Maeng) }]^{*} 100$.

De los resultados anteriores se evidencia que el Fuzzy Logic Toolbox de MATLAB es una herramienta con la cual se pueden obtener muy buenas aproximaciones de la remoción de compuestos estrogenicos, basándose en las reglas de inferencia borrosa construidas a partir de resultados experimentales y del conocimiento de expertos.

Las ligeras desviaciones pueden tener origen en que no hay suficientes reglas de inferencia para determinar una mejor salida a partir de las entradas, por lo que se debe hacer más robusto el sistema de control borroso. Para ello se deben agregar más reglas de inferencia borrosa, con lo que el sistema se hará mucho más inteligente (39).

Estas nuevas reglas de inferencia pueden salir de los resultados de otras investigaciones en donde se haya utilizado un BRM para la remoción de compuestos estrogénicos, y en donde el TRS haya sido estudiado para medir la influencia en la degradación de estos compuestos. 
Tabla 12. Comparación de la remoción (\%) de estrógenos

\begin{tabular}{|c|c|c|c|}
\hline \multicolumn{4}{|c|}{$\%$ RCE, E1 } \\
\hline BRM & $\begin{array}{c}\text { Maeng, et al. } \\
\text { (26) }\end{array}$ & $\begin{array}{c}\text { Toolbox Fuzzy } \\
\text { Logic }\end{array}$ & $\%$ Error \\
\hline $\mathrm{R} 1$ & 97.94 & 94.50 & 3.51 \\
\hline $\mathrm{R} 2$ & 100.00 & 100.00 & 0.00 \\
\hline $\mathrm{R} 3$ & 100.00 & 100.00 & 0.00 \\
\hline \multicolumn{4}{|c|}{$\%$ RCE, E2 } \\
\hline $\mathrm{R} 1$ & 97.85 & 94.50 & 3.42 \\
\hline $\mathrm{R} 2$ & 100.00 & 100.00 & 0.00 \\
\hline $\mathrm{R} 3$ & 100.00 & 100.00 & 0.00 \\
\hline \multicolumn{4}{|c|}{$\%$ RCE, EE2 } \\
\hline $\mathrm{R} 1$ & 39.50 & 39.50 & 0.00 \\
\hline $\mathrm{R} 2$ & 70.60 & 69.50 & 1.56 \\
\hline $\mathrm{R} 3$ & 78.10 & 85.00 & -8.83 \\
\hline
\end{tabular}

\section{CONCLUSIONES}

Se simuló la influencia del TRS en la remoción de estrógenos mediante control borroso, determinando que el sistema borroso diseñado es una muy buena aproximación para obtener resultados cercanos a los obtenidos mediante un sistema experimental real.

Para minimizar las desviaciones entre un método y otro es conveniente incluir más reglas de inferencia borrosa, a fin de que el motor del sistema pueda hacer un mayor número de inferencias, determinando una mejor salida a partir de las entradas.

\section{REFERENCIAS}

1. Nakada, N., Tanishima, T., Shinohara, H. y Kiri, K. Pharmaceutical chemicals and endocrine disrupters in municipal wastewater in Tokio and their removal during activated sludge treatment. Water Research. 2006;40:3297-3303.

2.Zorita, S., Martensson, L. y Mathiasson, L. Occurrence and removal of pharmaceutical sewage treatment system in the south of Sweden. Science of the Total Environment. 2009;407:2760-70. 3. Ternes, T., Stumpf, M., Mueller, J., Haberer, K., Wilken, R. D. y Servos, M. Behaviour and ocurrence of estrogens in municipal sewage treatment plants. 1: Investigación in Germany, Canada and Brazil. Science of the Total Environment. 1999;225: 81-90.

4. Baronti, Ch., Curini, R., D'Ascenso G., Di Corcia, A., Gentili, A. y Samperi, R. Monitoring natural and synthetic estrogens al activated sludge sewage treatment plants and in a receiving river water. Environmental Science and Technology, 2000;34(24):5059-66. 
5. Servos, M., Bennie, D., Burnison, B., Jurkovic, A., McInnis, R., Neheli, T., Schnell, A., Seto, P., Smyth, S. y Ternes, T. Distribution of estrogens, $17 \beta$-estradiol and estrone, in Canadian municipal wastewater treatment plants. Science of the Total Environment. 2005;336:155-70.

6. Liu, Z-H., Arogo, J., Prudem, A. y Knowlton, K. Occurrence, fate and removal of synthetic oral contraceptives (SOCs) in the natural environment: a review. Science of the Total Environment. 2011;409: 5149-61.

7. Vethaak, A., Lahr, J. y Kuiper, R. Estrogenic effects in fish in The Netherlands: Some Preliminary results. Toxicology. 2002;181:147-50.

8. Purdom, C., Hardiman, V., Eno, C., Tyler, C. y Sumpter, J. Estrogenic effects on effluents from sewage treatment Works. Chemical Ecology. 1994;8:275-85.

9. Petrovic, M., Solé, M., López de Alda, M. y Barceló, D. Endocrine disruptors in sewage plants, receiving river waters and sediments: Integration of chemical analysis and biological effects on feral carp. Environ. Toxicol. Chem. 2002;21:2146-56.

10. Solé, M., de Alda, M., Castillo, M., Porte, C., Ladegaard-Pedersen, K. y Barceló, D. Estrogenicity determination in sewage treatment plants in surface waters from the Catalonian area (NE Spain). Environmental Science and Technology. 2000;34:5076-83.

11. Forrez, I., Boon, N., Verstraete, W. y Carballa. M. Municipal Wastes Biodegradation of Micropollutants and Prospects for Water and Wastewater Biotreatment. In: Murray MooYoung (ed.), Comprehensive Biotechnology, Second Edition. 2011;6:485-94. Disponible en: http://www.usc.es/biogrup/sites/default/files/2011_Book_chapter-Forrez-Biodegradation_of_ micropollutants1.pdf. [Fecha de acceso: 02 de marzo de 2014].

12. Cóppola, F., Nader, J. y Aguirre, R. Metabolismo de los estrógenos endógenos. Rev. Med. Urug. 2005;21:15-22. http://www.scielo.edu.uy/pdf/rmu/v21n1/v21n1a03.pdf. [Fecha de acceso: 05 de marzo de 2014].

13. Martínez, S., Colomina, M., González, E., Moya, L., Redolat, R., Suay, F., Torenbeek, M. y Vicens, P. Hormonas, estado de ánimo y función cognitiva. Primera edición. España: Delta, Publicaciones Universitarias, 2007:233-5.

14. Guzmán, C. y Zambrano, E. Compuestos disruptores endocrinos y su participación en la programación del eje reproductivo. Revista de Investigación Clínica. 2007;59(1):73-81. Disponible en: http://scielo.unam.mx/pdf/ric/v59n1/v59n1a9.pdf. [Fecha de acceso: 28 de febrero de 2014]. 15. Lehmann, A., Cortacans, J., González, I., Hernández, A., Gil, J. y Martin, J. Eficacia de los tratamientos avanzados en la eliminación de sustancias estrogénicas. III Congreso de Ingeniería Civil, Territorio y Medio Ambiente, Agua, Biodiversidad e Ingeniería. Zaragoza, España, octubre 25 al 27 de 2006. 2006. Disponible en la siguiente dirección electrónica: http://www.ciccp.es/ biblio_digital/Icitema_III/congreso/pdf/010510.pdf. [Fecha de acceso: 07 de febrero de 2014]. 16. Xue, W., Wu, Ch., Xiao, K., Huang, X. y Zhou, H. Elimination and fate of selected microorganic pollutants in a full-scale anaerobic/anoxic/aerobic process combined with membrane bioreactor for municipal wastewater reclamation. Water Research. 2010; 44:5999-6010.

17. Melin, T., Jefferson, B., Bixio, D., Thoeye, C., De Wilde, W., De Koning, J., Van der Graaf, J. y Wintgens, T. Membrane bioreactor technology for wastewater treatment and reuse. Desalination. 2006; 187:271-82.

18. Till, S. y Mallia, H. Membrane bioreactors: wastewater treatment applications to achieve high quality effluent. 64th Annual Water Industry Engineers and Operators Conference, 5 and 6 September, 2001. 2001;57-65. Disponible en la dirección electrónica: http://d4801.mysite. westnethosting.com.au/conference_papers/2001/pdf/paper8.pdf. [Fecha de acceso: 27 de febrero de 2014].

19. Zuriaga, E. Estudio de la relación entre las características químicas del licor mezcla en biorreactores de membrana y su correlación con las resistencias a la filtración. [Tesis de Máster]. 
[España]. Universidad Politécnica de Valencia. 2010. Disponible en: http://riunet.upv.es/bitstream/ handle/10251/11417/Tesis\%20de\%20M\%C3\%A1ster_Elena\%20Zuriaga\%20Agust\%C3\%AD. pdf? sequence $=1$. [Fecha de acceso: 07 de marzo de 2014].

20. Torrescano, J. Parámetros de operación en el proceso de tratamiento de agua residual por lodos activados. Aquaforum. 2009;13(52):14-9. Disponible en: http://seia.guanajuato.gob. $\mathrm{mx} /$ document/AquaForum/AF52/AF5204_ParametrosOperacion.pdf. [Fecha de acceso: 04 de marzo de 2014].

21. Hu, J., Chen, X., Tao, G. y Kekred, K. Fate of Endocrine Disrupting Compounds in Membrane Bioreactor Systems. Environ. Sci. Technol. 2007;41:4097-102.

22. Lee, J., Lee, B., Ra, J., Cho, J., Kim, In S., Chang, N., Kim, H. y Kim, S. Comparison of the removal efficiency of endocrine disrupting compound in pilot scale sewage treatment processes. Chemosphere. 2008;71:1582-92.

23. Tadkaew, N., Hai, F., McDonald, J., Khan, S. y Nghiem, L. Removal of trace organics by MBR treatment: The role of molecular properties. Water Research. 2011;45:2439-51.

24. Roh, H. y Chu, K-H. Effects of solids retention time on the performance of bioreactors bioaumented with a $17 \beta$-estradiol-utilizing bacterium, Sphingomonas strain KC8. Chemosphere. 2011;84:227-33.

25. Nguyen, L., Hai, F., Kang, J., Price, W. y Nghiem, L. Removal of trace organic contaminants by a membrane bioreactor-granular activated carbon (MBR-GAC) system. Bioresource Technology. 2012;113:169-73.

26. Maeng, S., Choi, B., Lee, K. y Song, K. Influences of solid retention time, nitrification and microbial activity on the attenuation of pharmaceuticals and estrogens in membrane bioreactors. Water Research. 2013;47:3151-62.

27. Torres, E. Lógica Formal Postmoderna. Material con fines didácticos. Doctorado en Ciencias de la Ingeniería, mención Productividad. Barquisimeto: UNEXPO. 2013.

28. Guzmán, D. y Castaño, V. La Lógica difusa en ingeniería: principios, aplicaciones y futuro. Ciencia y Tecnología. 2006;24(2):87-107.

29. Manga, J., Serralta, J., Seco, A. y Ferrer, J. Desarrollo de un sistema de control basado en lógica difusa para la aireación en un proceso de fangos activados. Ingeniería \& Desarrollo, Universidad del Norte. 2001;9:35-56. Disponible en la dirección electrónica: http://www. redalyc.org/articulo.oa?id=85200904. [Fecha de acceso: 05 de marzo de 2014].

30. Marsili-Libelli, S. y Giunti, L. Fuzzy predictive control for nitrogen removal in biological wastewater treatment. Water Science and Technology. 2002;45(4):37-44.

31. Mohd, S., Khor, W. y Ya'acob, M. Fuzzy logic of a nonlinear pH-neutralisation in wastewater treatment plant. International Journal of Engineering and Technology. 2004;1(2):197-205.

32. Fiter, M., Colprin, J., Freixó, A. y Rodríguez-Roda, I. Control basado en lógica difusa para los sólidos en suspensión. Ingeniería Química. 2006;104-112.

33. Caraman, S., Sbarciog, M. y Barbu, M. Predictive Control of a Wastewater Treatment Process. International Journal of Computers, Communications \& Control. 2007;2(2):132-142.

34. Holenda, B., Domokos, E., Rédey, Á. y Fazakas, J. Dissolved oxygen control of the activated sludge wastewater treatment process using model predictive control. Computers and Chemical Engineering. 2008;32:1270-78.

35. Carlos-Hernández, S., Sánchez, E. y Beteau, J. Fuzzy Control Strategy for an AnaerobicWastewater Treatment Process. Chem. Biochem. Eng. Q. 2010;24(2):235-45.

36. Hernández, M. y Delgadillo, L. Control del flujo de metano en el tratamiento de aguas residuales industriales usando lógica fuzzy. El Hombre y la Maquina. 2012;38:22-8.

37. Khokhar, Z., Al-Harthi, M. y Bdurraheem, A. Investigations of Biosorption Capacity Histories with Temperature and Other Parameters in a Given Fuzzy Universe of Discourse. International Journal of Modeling and Optimization. 2012;2(3):222-6. 
38. Narváez, V. Modelamiento matemático del control de proceso de una planta de tratamiento de aguas residuales mediante lógica difusa utilizando MATLAB. Trabajo Especial de Grado. [Quito]. Universidad Politécnica Salesiana. 2013.

39. Ramdewor, P., Soneechur, V., Kishnah, S. y Pudaruth, S. Simulation of the nitrification process in wastewater treatment using fuzzy logic. 2nd International Conference on Machine Learning and Computer, August 25-26, 2013. Kuala Lampur, Malaysia. 2013.

40. Sánchez, L. Control borroso para la valoración del impacto ambiental generado por contaminantes emergentes en aguas residuales hospitalarias. Revista Gestión y Ambiente. 2015;18(1):81-93. 